\title{
Crosslinked Polymer Nanoassemblies and Their Delivery Applications
}

\author{
Zhao Wang, ${ }^{a}$ Filipe Olim, ${ }^{b}$ Ana Rute Neves, ${ }^{b}$ Mariana Vieira, ${ }^{b}$ Jingjing Sun, ${ }^{c}$ Helena Tomás, ${ }^{b}$ \\ and Ruilong Sheng ${ }^{\star, b}$ \\ ${ }^{a}$ School of Material Engineering, Jinling Institute of Technology, Nanjing, Jiangsu 211169, China \\ ${ }^{b}$ CQM - Centro de Química da Madeira, Universidade da Madeira, Campus da Penteada, 9000-390 Funchal, Portugal \\ ${ }^{c}$ Center for Pharmacogenetics, Department of Pharmaceutical Sciences, School of Pharmacy, University of Pittsburgh, \\ Pittsburgh, PA 15261, United States
}

Email: ruilong.sheng@staff.uma.pt (R. S.)

\begin{abstract}
Chemical crosslinking technology was rapidly developed and utilized to prepare structurally fixed, stabilized and functionalized polymer nanoassemblies and nanoarchitectures by covalently reinforcing the interactions between the polymer chains. This perspective reviewed recent advances on the crosslinked polymer nanoassemblies and their applications, mainly in drug delivery. Moreover, possible future research outlooks in the field of Shell-crosslinked polymer nanoassemblies were also stated and discussed.
\end{abstract}

Keywords crosslinked polymers, crosslinker, nanomaterials, controllable, applications

Functional polymer nanoassemblies with various morphologies (e.g., micelles, vesicles, rods, disks, etc.) showed great potential in therapeutics delivery, bioimaging, biosensors, bioactuators, bioelectronics, food packaging and nanodevices. ${ }^{[1]}$ However, majority of the polymer nanoassemblies were formed via non-covalent driven forces, which offers them dynamic features and results in low stability under the conditions such as dilution, change of $\mathrm{pH}$ value and ionic strength, thermal and specific chemical stimulators, largely limiting their biomedical applications. ${ }^{[2]}$ To develop stabilized polymer nanoassemblies, crosslinking technique, a covalent approach, was rapidly developed and utilized to prepare chemically fixed, stabilized and functionalized polymer assemblies/architectures by reinforcing the interactions between the polymer chains.

Up to date, a number of crosslinked polymers and crosslinking methods have been developed for the construction of crosslinked nanoassemblies. ${ }^{[3]}$ In this field, rational utilization of high efficient, economical, biocompatible, controllable and quantitative crosslinkers and related crosslinking strategies, scale-up production of the crosslinked polymer assemblies with good reproducibility still remain challenging. ${ }^{[4]}$ Deciphering of the correlation between the structure/architecture of crosslinked polymer nanoassemblies and their physico-chemical, environmental and biomedical properties is also important. Many works have been performed to explore the above challenging topics and try to overcome the technical bottlenecks and shortcomings. To date, shell-crosslinked, ${ }^{[5]}$ core-crosslinked $^{[6]}$ and interface-crosslinked ${ }^{[7]}$ nanoassembies had been developed as stabilized nanocarriers, mainly for drug delivery. Among them, shell-crosslinked nanoassembies take the advantages of: (1) comparatively large and flexible hydrophobic core which could enhance drug loading capacity; ${ }^{[8]}$ (2) hydrophilic polymer shell that could be functionalized by stimuli-responsive crosslinkers and make the nanoassemblies "smart". [9] Acetal-crosslinker-containing shell-crosslinked PEG-PAsp-PPhe nanomicelles showed high DOX-loading capacity, good stability under the existence of sodium dodecyl sulfate (SDS), and $\mathrm{pH}$-responsive DOX release. ${ }^{[10]}$ Cystamine could be used as a crosslinker to react with
$\mathrm{N}$-acryloxysuccinimide (NAS)-containing polymer to prepare Shell-crosslinked PEO-b-P(DMA-stat-NAS)- $b$-PNIPAM micelles with high solution stability and the micelles showed bioreduction-responsive properties. ${ }^{[1]}$ By visible light-induced diselenide metathesis, CPT and DOX co-loaded shell crosslinked micelles ${ }^{[12]}$ were prepared and they demonstrated controllable CPT/DOX dual drug release manners in tumor microenvironments. Apart from these $\mathrm{pH}$, redox and photo-responsive systems, ROS responsive ${ }^{[13]}$ protease and hyaluronidase responsive ${ }^{[14]}$ shell-crosslinked nanoassemblies were also developed. Towards the application of nanoassemblies under multiple stimulants, some shell-crosslinked dual responsive nanoassemblies were developed. Redox/pH dual responsive PCL- $b$-P(OEGMA-coMAEBA and folic acid-modified micelles were prepared for camptothethin (CPT) delivery by using disulfide-bond and hydrazone linkage as the crosslinker, the loaded CPT micelles exhibited accelerated CPT release under low $\mathrm{pH}$ value and/or redox agent dithiothreitol (DTT). ${ }^{[15]}$ Our group recently developed a series of in-situ shell-crosslinked PDPA- $b$ $\mathrm{P}$ (NMS-co-OEG) diblock terpolymer micelles with $\mathrm{pH}$-sensitive diisopropylamine and cystamine crosslinker, the micelles demonstrated remarkable $\mathrm{pH} /$ redox-dual responsive manners for Camptothecin (CPT) delivery application. ${ }^{[16]}$ Although these progresses had been achieved, rationally in-situ incorporate crosslinkers into nanoassemblies to achieve low cytotoxicity, high drug loading efficiency, as well as dual, triple or multiple-stimuli responsive delivery manners is still a big challenge. Moreover, the structural characterization for the shell-crosslinked multi-stimuli responsive micelles needs to be further investigated. The construction of various functional shell-crosslinked polymer nanoassemblies is illustrated in Scheme 1.

Regarding the future innovation of crosslinked polymers, there are several issues to be extensively studied: (1) developing green, efficient, controllable and modular methods to prepare novel crosslinkable functional polymers with sustainability, ${ }^{[17]}$ controlled release properties under physicochemical stimuli-factors (thermal, light, mechanical, magnetic, 
Scheme 1 Preparation of shell-crosslinked polymer nanoassemblies by in situ crosslinking methodology

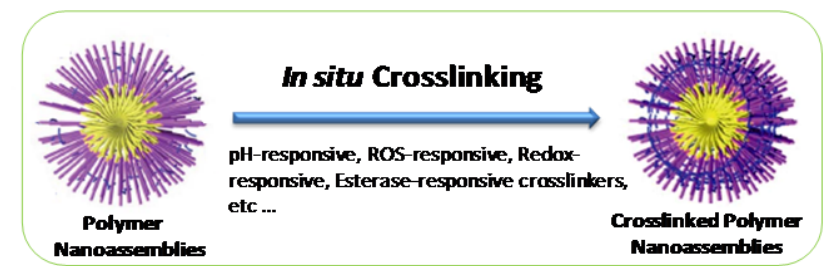

Scheme 2 Future perspective of crosslinked polymer and their nanoassemlies

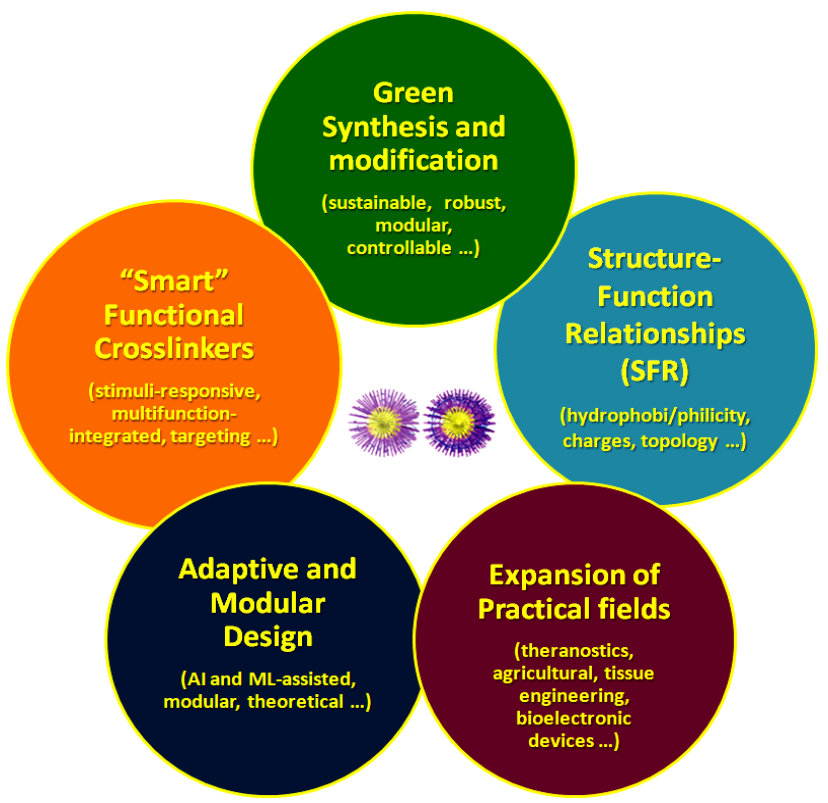

electrical and radiation, molecular/biomolecular) $;^{[18]}$ (2) expanding the structure/function diversity of the "smart" (reversible, stimuli-responsive, multifunction-integrated, biotargeting, etc.) organic crosslinker molecules, and macromolecular/dendrimer crosslinkers via clickable, controllable technique, ${ }^{[19]}$ (3) elucidating the structure-function relationship (SFR), especially the correlation between crosslinkers and nanoassemblies' particle size and morphology, solution stability, stimuli-responsive feature, cytotoxicity, cellular uptake, intracellular behaviors, as well as therapeutic effects; (4) Based on the evaluation data, combining with artificial intelligence $(\mathrm{Al})$ and machine learning $(\mathrm{ML})$ to optimize the crosslinked nanobiomaterials to reach the desired performance by adaptive and modular-based design; (5) expanding the practical applications of crosslinked polymer nanoassemblies in the realms such as drug/gene/biomolecules delivery, agrochemicals/fragrant/nutraceutical encapsulation, nanomaterial-assisted tissue engineering and in vivo delivery, as well as bioelectronic devices-based smart delivery. The above-mentioned areas need to be systematically addressed (Scheme 2).

\section{Acknowledgement}

Dr. Ruilong Sheng thanks FCT-Fundação para a Ciência e a Tecnologia (project UIDB/00674/2020, and Programmatic Fund -UIDP/00674/2020, CQM, Portuguese Government funds), ARDITI-Agência Regional para o Desenvolvimento da Investigação Tecnologia e Inovação, through the project M1420-01-0145-FEDER-000005-Centro de Química da Madeira-CQM ${ }^{+}$(Madeira 14-20) and ARDITI-CQM-2017-ISG-
003 ARDITI-CQM-2017-PDG-009 and ARDITI-2017-PDG-011 for sponsorship. Dr. Zhao Wang thanks the fundation support from Jinling Institute of Technology (No. jit-b-201828) and the Natural Science Foundation of Jiangsu Province (No. BK20190113).

\section{Conflict of Interest}

The authors declare no conflict of interest.

Copyright (C) 2021 Zhao Wang, Filipe Olim, Ana Rute Neves, Mariana Vieira, Jingjing Sun, Helena Tomás, and Ruilong Sheng. This article is an open access article distributed under the terms and conditions of the Creative Commons Attribution (CC BY) license (http://creativecommons. org/licenses/by/4.0/). The use, distribution or reproduction in other forums is permitted, provided the original author(s) or licensor are credited and that the original publication in this journal is cited, in accordance with accepted academic practice. No use, distribution or reproduction is permitted which does not comply with these terms.

\section{References}

[1] (a) Rahim, M. A.; Kristufek, S. L.; Pan, S.; Richardson, J. J.; Caruso, F. Phenolic Building Blocks for the Assembly of Functional Materials. Angew. Chem. Int. Ed. 2019, 58, 1904-1927; (b) Chen, L.; Yan, C.; Zheng, Z. Functional polymer surfaces for controlling cell behaviors. Mater. Today 2018, 21, 38-59.

[2] D'Agosto, F.; Rieger, J.; Lansalot, M. RAFT-Mediated Polymerization-Induced Self-Assembly. Angew. Chem. Int. Ed. 2020, 59, 8368-8392.

[3] (a) Azeredo, H. M. C.; Waldron, K. W. Crosslinking in polysaccharide and protein films and coatings for food contact $-\mathrm{A}$ review. Trends Food Sci. Technol. 2016, 52, 109-122; (b) Vanherck, K.; Koeckelberghs, G.; Vankelecom, I. F. J. Crosslinking polyimides for membrane applications: A review. Prog. Polym. Sci. 2013, 38, 874-896; (c) Parhi, R. Cross-Linked Hydrogel for Pharmaceutical Applications: A Review. Adv. Pharm. Bull. 2017, 7, 515-530.

[4] Reddy, N.; Reddy, R.; Jiang, Q. Crosslinking biopolymers for biomedical applications. Trends Biotechnol. 2015, 33, 362-369.

[5] (a) Thurmond, K. B.; Kowalewski, T.; Wooley, K. L. Water-soluble knedel-like structures: the preparation of shell-cross-linked small particles. J. Am. Chem. Soc. 1996, 118, 7239-7240; (b) Thurmond, K. B.; Kowalewski, T.; Wooley, K. L. Shell cross-linked knedels: A synthetic study of the factors affecting the dimensions and properties of amphiphilic core-shell nanospheres. J. Am. Chem. Soc. 1997, 119, 6656-6665.

[6] Cheng, Y.; He, C.; Xiao, C.; Ding, J.; Ren, K.; Yu, S.; Zhuang, X.; Chen, $X$. Reduction-responsive cross-linked micelles based on PEGylated polypeptides prepared via click chemistry. Polym. Chem. 2013, 4, 3851-3858.

[7] Dai, J.; Lin, S.; Cheng, D.; Zou, S.; Shuai, X. InterlayerCrosslinked Micelle with Partially Hydrated Core Showing Reduction and pH Dual Sensitivity for Pinpointed Intracellular Drug Release. Angew. Chem. Int. Ed. 2011, 50, 9404-9408.

[8] (a) Baugher, A. H.; Goetz, J. M.; McDowell, L. M.; Huang, H.; Wooley, K. L.; Schaefer, J. Location of fluorotryptophan sequestered in an amphiphilic nanoparticle by rotational-echo double-resonance NMR. Biophys. J. 1998, 75, 2574-2576; (b) Kao, H.-M.; Stefanescu, A. D.; Wooley, K. L.; Schaefer, J. Location of terminal groups of dendrimers in the solid state by rotational-echo double-resonance NMR. Macromolecules 2000, 33, 6214-6216; (c) Kao, H.-M.; O'Connor, R. D.; Mehta, A. K.; Huang, H.; Poliks, B.; Wooley, K. L.; Schaefer, J. Location of cholic acid sequestered by core-shell nanoparticles using REDOR NMR. Macromolecules 2001, 34, 544-546.

[9] (a) Davis, K. A.; Anseth, K. S. Controlled release from crosslinked 
degradable networks. Critical Reviews ${ }^{\mathrm{TM}}$ in Therapeutic Drug Carrier Systems 2002, 19, DOI: 10.1615/CritRevTherDrugCarrierSyst.v19.145.30; (b) Murthy, K. S.; Ma, Q.; Clark Jr., C. G.; Remsen, E. E.; Wooley, K. L. Fundamental design aspects of amphiphilic shell-crosslinked nanoparticles for controlled release applications. Chem. Commun. 2001, 773-774.

[10] Lee, S. J.; Min, K. H.; Lee, H. J.; Koo, A. N.; Rim, H. P.; Jeon, B. J.; Jeong, S. Y.; Heo, J. S.; Lee, S. C. Ketal cross-linked poly (ethylene glycol)-poly (amino acid)s copolymer micelles for efficient intracellular delivery of doxorubicin. Biomacromolecules 2011, 12, 1224-1233.

[11] Li, Y.; Lokitz, B. S.; Armes, S. P.; McCormick, C. L. Synthesis of reversible shell cross-linked micelles for controlled release of bioactive agents. Macromolecules 2006, 39, 2726-2728.

[12] Zhai, S.; Hu, X.; Hu, Y.; Wu, B.; Xing, D. Visible light-induced crosslinking and physiological stabilization of diselenide-rich nanoparticles for redox-responsive drug release and combination chemotherapy. Biomaterials 2017, 121, 41-54.

[13] Deepagan, V. G.; Kwon, S.; You, D. G.; Nguyen, V. Q.; Um, W.; Ko, H.; Lee, H.; Jo, D.-G.; Kang, Y. M.; Park, J. H. In situ diselenide-crosslinked polymeric micelles for ROS-mediated anticancer drug delivery. Biomaterials 2016, 103, 56-66.

[14] Ekkelenkamp, A. E.; Elzes, M. R.; Engbersen, J. F. J.; Paulusse, J. M. J. Responsive crosslinked polymer nanogels for imaging and therapeutics delivery. J. Mater. Chem. B 2018, 6, 210-235.

[15] (a) Hu, X.; Li, H.; Luo, S.; Liu, T.; Jiang, Y.; Liu, S. Thiol and pH dual-responsive dynamic covalent shell cross-linked micelles for triggered release of chemotherapeutic drugs. Polym. Chem. 2013, 4, 695-706; (b) Hu, X.; Tian, J.; Liu, T.; Zhang, G.; Liu, S. Photo-triggered release of caged camptothecin prodrugs from dually responsive shell cross-linked micelles. Macromolecules 2013, 46, 6243-6256.

[16] Sun, J.; Wang, Z.; Cao, A.; Sheng, R. Synthesis of crosslinkable diblock terpolymers PDPA- $b$-P(NMS-Co-OEG) and preparation of shell-crosslinked $\mathrm{pH} /$ redox-dual responsive micelles as smart nanomaterials. RSC Adv. 2019, 9, 34535-34546.

[17] Balalaie, A.; Rezvani, M. B.; Mohammadi Basir, M. Dual function of proanthocyanidins as both MMP inhibitor and crosslinker in dentin biomodification: A literature review. Dent. Mater. J. 2018, 37, 173-182.

[18] (a) Ligon-Auer, S. C.; Schwentenwein, M.; Gorsche, C.; Stampfl, J.; Liska, R. Toughening of photo-curable polymer networks: a review. Polym. Chem. 2016, 7, 257-286; (b) Dai, Y.; Chen, X.; Zhang, X., Recent advances in stimuli-responsive polymeric micelles via click chemistry. Polym. Chem. 2019, 10, 34-44; (c) Mejías, J. C.; Roy, $\mathrm{K}$, In-vitro and in-vivo characterization of a multi-stage enzyme-responsive nanoparticle-in-microgel pulmonary drug delivery system. J. Control. Release 2019, 316, 393-403.

[19] (a) Li, N.; Cai, H.; Jiang, L.; Hu, J.; Bains, A.; Hu, J.; Gong, Q.; Luo, K.; Gu, Z. Enzyme-Sensitive and Amphiphilic PEGylated Dendrimer-Paclitaxel Prodrug-Based Nanoparticles for Enhanced Stability and Anticancer Efficacy. ACS Appl. Mater. Interfaces 2017, 9, 6865-6877; (b) Krishnakumar, G. S.; Sampath, S.; Muthusamy, S.; John, M. A. Importance of crosslinking strategies in designing smart biomaterials for bone tissue engineering: A systematic review. Mater. Sci. Eng.: C 2019, 96, 941-954; (c) Wang, Z.; Olim, F.; Sun, J.; Neves, A. R.; Mendes, F.; Tomás, H.; Sheng, R. Prodrug Systems (II): A Perspective of Polymer-Based Doxorubicin Prodrug Systems towards Chemotherapy. Med. Res. 2021, 5, 200023; (d) Wang, Z.; Neves, A. R.; Olim, F.; Tomás, H.; Tang, S.; Sheng, R. Green Polymers toward Nanobiotechnology(I): Synthesis of Glycopolypeptides and Their Analogues. Gen. Chem. 2019, 5, 190010.

Received June 12, 2021 Accepted June 20, 2021 